\title{
THE HAYMAN-WU CONSTANT
}

\author{
KNUT ØYMA
}

(Communicated by Clifford J. Earle, Jr.)

Abstract. The Hayman-Wu constant is at least $\pi^{2}$.

Let $D$ be the open unit disc and $T$ its boundary. The length of a curve $K$ is denoted $|K|$. The Hayman-Wu theorem says that there is a constant $C$ such that if $f(z)$ is univalent in $D$ and $L$ is any line then $\left|f^{-1}(L)\right| \leq C$ (see [3]). The Hayman-Wu constant is the least possible value of $C$. Its numerical value is unknown, but in [4] it is proved that $C \leq 4 \pi$. It has been conjectured that $C=8 \int_{0}^{1} d x / \sqrt{1+x^{4}}$ (see [1]); however, we will prove

Theorem. $C \geq \pi^{2}$.

Flinn proved in [2] that if $f(D)$ contains one component of $\mathbb{C} \backslash L$ then $\left|f^{-1}(L)\right| \leq \pi^{2}$. Our example shows that this is the best possible result in this case. The proof uses an elementary fact about harmonic measure: If $I$ is a subarc of $T$ and $0<c<1$ then the level curve $\omega(z, I, D)=c$ is a circular arc through the endpoints of $I$ meeting $T \backslash I$ at an angle of $c \pi$.

Let $\Pi^{+}$and $\Pi^{-}$be the upper and lower half planes respectively. If $I$ is an interval of the real line and $0<\varepsilon<1$ then let $C_{I, \varepsilon}$ be the circle centered in $\Pi^{+}$meeting $\mathbb{R}$ at the endpoints of $I$ such that the (least) angle between $C_{I, \varepsilon}$ and $\mathbb{R}$ is $\varepsilon$. We define $C_{I, \varepsilon} \cap \Pi^{+}=S_{I, \varepsilon}$. Let $\Omega_{I, \varepsilon}$ be the unbounded component of $\mathbb{C} \backslash \overline{\left(S_{I, \varepsilon} \cup S_{I, \varepsilon / 2}\right)}$. Two lemmas are needed.

Lemma 1. For $z \in I, \omega(z)=\omega\left(z, S_{I, \varepsilon}, \Omega_{I, \varepsilon}\right)<\frac{1}{2}+\varepsilon$.

Proof. Without loss of generality $I$ equals $[0,1]$. If we use the transformation $g(z)=1 / z-1$, we may assume that $\Omega_{I, \varepsilon}=\left\{r e^{i \phi}: r>0,-\pi+\varepsilon<\phi<\pi+\varepsilon / 2\right\}$ and that $I=\mathbb{R}^{+}$. Then $\omega(z)$ is given by the formula

$$
\omega\left(r e^{i \phi}\right)=(\pi+\varepsilon / 2-\phi) /(2 \pi-\varepsilon / 2) .
$$

Therefore, $\omega(z)=(\pi+\varepsilon / 2) /(2 \pi-\varepsilon / 2)<\frac{1}{2}+\varepsilon$ for $z \in \mathbb{R}^{+}$.

Lemma 2. For every $\delta>0$ there exist numbers $b>0$ and $\varepsilon>0$ such that if $I$ is a subarc of $T$ of length less than $b$ and $K$ is a crosscut in $D$ connecting

Received by the editors October 3, 1991 and, in revised form, January 17, 1992.

1991 Mathematics Subject Classification. Primary 30C85.

Key words and phrases. Harmonic measure, conformal mapping. 
the endpoints of $I$ satisfying $\omega(z, I, D)<\frac{1}{2}+\varepsilon$ for every $z \in K$, then $|K|>$ $|I|(1-\delta) \pi / 2$.

Proof. $K$ lies outside the convex curve $\omega(z, I, D)=\frac{1}{2}+\varepsilon$. If $|I|$ and $\varepsilon$ are small then this curve is almost a half circle whose diameter is almost $|I|$. A routine but tedious calculation shows that

$$
\left|\omega(z, I, D)=\frac{1}{2}+\varepsilon\right|>(\sin (|I| / 2))(\pi-|I|-2 \varepsilon \pi) .
$$

Proof of the theorem. If $\delta>0$ choose $\varepsilon$ as in Lemma 2. Define $I_{0}^{1}=[0,1]$ and $d=\operatorname{diam}\left(C_{I_{0}^{1}, \varepsilon / 2}\right)$. For $k \in Z$ let $I_{k}^{1}=I_{0}^{1}+2 k d$. The circles $C_{I_{k}^{1}, \varepsilon / 2}$ are disjoint. Let $\mathbb{R} \backslash \bigcup I_{k}^{1}=\bigcup J_{m}^{1}$, where the intervals $J_{k}^{1}$ are disjoint. Choose closed intervals $I_{n}^{2} \subset \bigcup J_{k}^{1}$ such that:

(i) $S_{I_{m}^{2}, \varepsilon / 2} \cap S_{I_{n}^{2}, \varepsilon / 2}=\varnothing$ for $m \neq n$;

(ii) $S_{I_{m}^{2}, \varepsilon / 2} \cap S_{I_{n}^{1}, \varepsilon / 2}=\varnothing$ for all $m, n$;

(iii) Each compact subset of $\mathbb{C}$ intersects only finitely many $I_{k}^{2}$;

(iv) $\left|\bigcup I_{k}^{2} \cap J_{m}^{1}\right|>\left|J_{m}^{1}\right| / 3 d$ for all $m$.

We can obtain (iv) by choosing each $I_{k}^{2}$ small. Let $\mathbb{R} \backslash\left(\bigcup I_{m}^{2} \cup I_{n}^{1}\right)=\bigcup J_{m}^{2}$. Continue the construction inductively.

Renumber the set $\left\{I_{m}^{k}\right\}=\left\{I_{n}\right\}$. Define $S_{n}=S_{I_{n}, \varepsilon}$ and let $O_{n}$ be the inside of $C_{I_{n}, \varepsilon}$. Define $\Omega=\left(\bigcup O_{n}\right) \cup \Pi^{-}$. The domain $\Omega$ is simply connected and the boundary of $\Omega$ equals $\left(\bigcup S_{n}\right) \cup E$ where $E \subset \mathbb{R}$. This is a Jordan arc, which is locally rectifiable since $\left|S_{n}\right| /\left|I_{n}\right|=$ constant. Therefore $\omega(z, E, \Omega) \equiv 0$ since $|E|=0$ by (iv). It follows that if $f(z)$ maps $D$ conformally onto $\Omega$ then $\sum\left|f^{-1}\left(S_{n}\right)\right|=2 \pi$.

By comparison $\omega_{n}(z)=\omega\left(z, S_{n}, \Omega\right)<\omega\left(z, S_{n}, \Omega_{I_{n}, \varepsilon}\right)$. Therefore, by Lemma $1, \omega_{n}(z)<\frac{1}{2}+\varepsilon$ for $z \in I_{n}$. Choose $f(z)$ such that $f(0)=-i a$ where $a$ is so large that $\omega_{n}(-i a)<b$ for all $n$. The constant $b$ comes from Lemma 2. $f^{-1}\left(I_{n}\right)$ is a crosscut in $D$ connecting the endpoints of $f^{-1}\left(S_{n}\right)$. Lemma 2 shows that $\left|f^{-1}\left(I_{n}\right)\right|>\left|f^{-1}\left(S_{n}\right)\right|(1-\delta) \pi / 2$. This proves the theorem since

$$
\left|f^{-1}(\mathbb{R})\right|=\sum\left|f^{-1}\left(I_{n}\right)\right| \geq \sum\left|f^{-1}\left(S_{n}\right)\right|(1-\delta) \pi / 2=\pi^{2}(1-\delta) .
$$

Conjecture. $C=\pi^{2}$.

\section{ACKNOWLEDGMENT}

I want to thank the referee for suggesting a better proof of Lemma 1.

\section{REFERENCES}

1. J. Fernandez, J. Heinonen, and O. Martio, Quasilines and conformal mappings, J. Analyse Math. 52 (1989), 117-132.

2. B. Flinn, Hyperbolic convexity and level sets of analytic functions, Indiana Univ. Math. J. 32 (1983), 831-841.

3. W. K. Hayman and J. M. G. Wu, Level sets of univalent functions, Comment. Math. Helv. 56 (1981), 366-403.

4. K. Øyma, Harmonic measure and conformal length, Proc. Amer. Math. Soc. 115 (1992). 687-690. 